\title{
REVISIÓN DE LA AUTOCARACTERIZACIÓN: UNA TÉCNICA NARRATIVA CONSTRUCTIVISTA
}

\author{
REVIEW OF SELF-CHARACTERIZATION: \\ A CONSTRUCTIVE NARRATIVE TECHNIQUE
}

\author{
JAVIER GONZÁLEZ-ENCINAS ${ }^{1}$, LUIS ÁNGEL SAÚL ${ }^{1}$ Y \\ JESÚS GARCÍA-MARTÍNEZ ${ }^{2}$
}

Cómo referenciar este artículo/How to reference this article:

González-Encinas, J., Saúl, L. A. y García-Martínez, J. (2019). Revisión de la autocaracterización: una técnica narrativa constructivista [Review of Self-characterization: A Constructive Narrative Technique ]. Acción Psicológica, 16(1), 105-128. https://doi.org/10.5944/ap.16.1.22192

\begin{abstract}
Resumen
La autocaracterización es una técnica narrativa enmarcada dentro de la Teoría de los Constructos Personales (Kelly, 1955). Se diferencia de otras técnicas narrativas por la estructura de sus instrucciones, donde se solicita a la persona describirse a sí misma en tercera persona de una forma íntima; y por el formato de corrección, que trata de desenfocar al lector de su visión y asumir el punto de vista del autor como propio, así como por explorar el proceso y el sistema de construcción
\end{abstract}

personal. Pese a ser destacada por su utilidad clínica (G. J. Neimeyer, 1996), no se han encontrado trabajos que reúnan los principales resultados que ha cosechado esta técnica. El objetivo de la presente investigación es analizar las similitudes y las diferencias encontradas en los trabajos que hacen uso de ella. Para dar respuesta, se lleva a cabo una revisión de literatura que reúne las principales contribuciones sobre la autocaracterización. Se ha tenido en cuenta la metodología empleada en los estudios, el formato de instrucción y de corrección y las etapas de análisis. La investigación muestra que el acercamiento crédulo, la flexibilidad de las instrucciones y la exploración de los constructos personales son los puntos

Correspondence address [Dirección para correspondencia]: Luis Ángel Saúl Gutiérrez. Universidad Nacional de Educación a Distancia (UNED), España. Email: lasaul@psi.uned.es

ORCID: Javier González Encinas (http://orcid.org/0000-0003-4026-8610), Luis Ángel Saúl Gutiérrez (http://orcid.org/0000-0002-6351-8283) y Jesús García Martínez (http://orcid.org/0000-0002-2698-6758).

${ }^{1}$ Universidad Nacional de Educación a Distancia (UNED), España.

${ }^{2}$ Universidad de Sevilla, España.

Recibido: 1 de mayo de 2018.

Aceptado: 17 de julio de 2018. 
recurrentes en los distintos trabajos recopilados. Se concluye que el formato de corrección, escasamente sistematizado, está influyendo negativamente en su uso y en su difusión a nivel empírico. Adicionalmente, se indica la necesidad de generar un protocolo de análisis que integre las principales etapas de análisis y actitudes del terapeuta que más atención han cosechado.

Palabras clave: Autocaracterización; Narrativa; Revisión; Teoría de los Constructos Personales.

\begin{abstract}
Self-characterization is a narrative technique framed in Personal Construct Theory (PCT; Kelly, 1955). It is differentiated from others narrative techniques by its elicited instructions, where is request a third person selfdescription in a sympathetic and intimate way, and by analysis protocol, that try to blur the reader from his own vision and assume the author's point of view as his own, like so explore the process and the system of personal construct. Despite being highlighted for therapeutic useful (G. J. Neimeyer, 1996), no works founds that gather the self-characterizations finds. The objective is analyzing the differences and similarities founded between research which use self-characterization. For give an answer, it is made a literature review that group the main contributions about this technique. It has been taken in consideration the methodology, instructions and analysis format, and analysis phases. This paper sample that credulous approach, instruction flexibility and personal construct exploration are the recurrent point in compiled works. It is been concluded that analysis format, poorly systematized, supposes a negative influence in its use and diffusion at empiric level. Additionally, it is indicated the need to generate a new protocol to analysis that integrated the principal phases and therapeutic attitude that have garnered more attention.
\end{abstract}

Keywords: Self-characterization; Narrative; Review; Personal Construct Theory.

\section{Introducción}

La autocaracterización es una técnica de evaluación constructivista que explora los constructos personales mediante un formato narrativo. Fue propuesta originalmente por Kelly (1955) y su expresión formal consiste en solicitar a la persona que va a ser evaluada una descripción de sí misma en tercera persona, es decir, desde la perspectiva de un observador. Este observador debe hacer la descripción desde un punto de vista de alguien que aprecie y conozca muy bien a la persona que se pretende describir. El texto generado es una narrativa, donde la persona se "autocaracteriza".

Una narrativa puede ser definida como una historia que se emplaza en un marco espacio-temporal concreto en el que se desarrolla la acción o un conjunto de acciones llevadas a cabo por un protagonista. Toda narrativa tiene un carácter intencional, trata de cambiar estados mentales tanto en el emisor como en el receptor del mensaje (García-Martínez, 2012). Los elementos estructurales que componen una narrativa y que permiten diferenciarla de otros géneros literarios son: contexto de la acción, suceso desencadenante, meta, consecuencias, cristalización del cambio y la presencia del protagonista.

La caracterización del protagonista es una de las características básicas de diferentes narrativas como el retrato, la autobiografía o la historia de vida (Beach, 1977). Ésta permite establecer diferencias entre el conjunto de personajes que pueblan el relato. De la misma manera, la RAE (2014) define caracterizar como: "determinar los atributos particulares de alguien o de algo, de modo que claramente se distinga de los demás" o "dicho de un actor: representar su papel con la verdad y fuerza de expresión necesarias para reconocer al personaje representado".

Distintos trabajos emplean el término de caracterización o caracterización del yo. En la mayoría de los casos, se describen perfiles psicopatológicos (Müller, Beutel y Wölfling, 2014) o perfiles de personalidad (Farkas y Orosz, 2013); en otros casos, se describen categorías de contenido temático (Lindquist y Barrett, 2008) y también se plantean como descripciones narrativas que sirven para obtener información del yo sin un esquema prefijado (McLean, 2015; Traen y Sorensen, 2008; Stephenson, 
Laszlo, Ehmann, Robert M. H. Lefever y Robin Lefever, 1997). George A. Kelly (1955) para generar su técnica de autocaracterización, se inspiró en el psicodrama de Moreno (1995). Moreno entiende que una de las metas que debe llevar a cabo el protagonista consiste en diferenciarse del resto y ponerse en la piel del otro (Masini, 2015). Por otro lado, en la representación teatral se abre un proceso de negociación donde el protagonista elige los caminos, vivencias $\mathrm{y}$ significados que va a transitar en su interpretación. La autocaracterización integra ambas características, la diferenciación del yo respecto al resto y la característica genuina de un personaje representando una obra.

Fruto de esta integración, la autocaracterización ha servido como una herramienta terapéutica al amparo de la técnica rol fijo. En dicha técnica, terapeuta y cliente generan un nuevo rol a partir del estudio de los constructos sugeridos en la autocaracterización y que posteriormente son puestos en práctica de forma intensiva durante un periodo acordado de tiempo en el mundo social del cliente (Kelly, 1955; R. A. Neimeyer et al., 2003), buscando ampliar o reducir la propia visión que cliente tiene sobre el sí mismo y el mundo que le rodea. Sin embargo, la autocaracterización presenta un conjunto de características muy específicas, a modo de instrucciones y de corrección que la diferencian del resto de técnicas narrativas.

\section{¿Qué es la autocaracterización, cuál su fundamento teórico y para qué sirve?}

La autocaracterización es una técnica que focaliza al autor en unos marcos discursivos concretos. Según Kelly (1955), las instrucciones habrían sido sometidas a una fuerte revisión y estaban cuidadosamente seleccionadas. La instrucción clásica dicta:

Quiero que escriba una descripción del personaje (nombre de la persona), como si fuese el protagonista de una obra teatral. Escríbalo como lo haría un amigo que le conoce profundamente y le comprende muy bien, quizás mejor que nadie lo haya hecho nunca. Asegúrese de escribirlo en tercera persona. Por ejemplo, puede comenzar diciendo, (nombre de la persona) es... (Kelly, 1955, p. 242, traducido por los autores).

Dichas instrucciones solicitan algo más que la descripción de la propia apariencia (Denicolo, 2003). Kelly pretendía con estas instrucciones que el individuo no se centrase en una descripción de fallos, sino en una descripción de la totalidad de su persona (Crittenden y Ashkar, 2012). Se intentaba que la persona expresase sus propios puntos de vista, más allá de una versión objetiva, sintiéndose libre de la amenaza que acompaña a la caracterización del yo.

Su objetivo principal es la descripción del sí mismo. Mantiene la característica de intencionalidad propia de las narrativas, pero no necesariamente contempla el resto de elementos estructurales que definen a éstas. Sin embargo, organiza un relato estructurado en torno a una temática, el sentido auto-percibido del yo.

La autocaracterización se enmarca dentro del enfoque constructivista de la Teoría de los Constructos Personales (TCP; Kelly, 1955). Con su uso se pretende explorar cómo las personas estructuran el mundo, cómo anticipan los acontecimientos a través de dicha estructura y dónde se localizan las personas respecto a sí mismas y el mundo (Kelly, 1955). La TCP se articula a partir de un postulado fundamental que establece que "los procesos de una persona se canalizan psicológicamente por las formas en las que anticipa los acontecimientos" (Botella y Feixas, 1998, p. 39; Kelly, 1955, p. 32) y 11 corolarios que se deducen del postulado inicial.

Dentro de la TCP, la unidad más relevante de exploración y que da origen al nombre de esta teoría, es el constructo personal. Un constructo personal es una dimensión evaluativa bipolar que se aplica, al menos, a dos elementos dados (personas, objetos, conceptos) y que permite establecer diferencias entre ellos a lo largo de esa dimensión según la propia experiencia. Ese contraste es el que da sentido al elemento dentro de un contexto, de modo que no hay adscripción de significado sin la diferencia entre los elementos. Por ejemplo, si algo es definido como flamenco, lo es en contraposición al elemento que se ubica en el otro polo del mismo constructo, así no son lo mismo los constructos flamenco-tango, flamenco-águila o flamenco-valón, constituyendo constructos completamente 
distintos. Estas dimensiones bipolares pueden ser verbales o no verbales (B. Green, 2004; Kelly, 1955) y se agrupan en un sistema de construcción personal que permite establecer relaciones jerárquicas entre los constructos. Las personas eligen aquel polo del constructo con el que anticipan una mayor capacidad definitoria del sistema (Botella y Feixas, 1998; Kelly, 1955).

En la autocaracterización se invita al individuo a explorar su sistema de construcción y a emplear aquellos constructos con los que más se identifica. Se puede usar para encontrar las áreas susceptibles de mejora durante el tratamiento clínico, ya que las técnicas psicométricas no permiten identificar la propia proyección del individuo. Según Kelly (1955), la autocaracterización permitía establecer hipótesis de partida, donde se reconociesen los recursos del individuo para modificar su propio sistema de construcción personal. Además, ha sido usada en diferentes fases del proceso terapéutico, tanto en etapas iniciales, como la diagnóstica, o finales, como la de seguimiento (Hardison y R. A. Neimeyer, 2007), así como para mejorar la alianza terapéutica (Crittenden y Ashkar, 2012).

En su origen, la autocaracterización estuvo ligada a su uso dentro de la técnica del rol fijo. Sin embargo, permite extraer datos y significados por sí misma, como demuestra el formato de análisis del protocolo propuesto por Kelly.

\section{¿Cómo se analiza una autocaracteri- zación?}

Los elementos que caracterizan a esta técnica y que la diferencian de otras técnicas narrativas son el formato de las instrucciones y el tipo de análisis de la exposición narrativa (o del texto).

En relación a la corrección de la técnica, Kelly abogaba por el mantenimiento de una actitud crédula ante el relato del individuo. No se trata de buscar una realidad objetiva que clasificar, sino adoptar el punto de vista del autor, el eslogan es «el cliente siempre tiene la razón». Podría darse el caso de que en una autocaracterización el relato del autor no estuviese sustentado en una experiencia fidedigna. Ante estas situaciones, siguiendo el principio del acercamiento crédulo, el autor nos estaría hablando de un posible cambio, ya que habría una diferencia entre lo que cuenta y en lo que se está intentando convertir (Jackson, 1988).

El análisis de la autocaracterización ha seguido un protocolo elaborado por Kelly (1955) con diferentes fases, en las que se emplean diferentes técnicas.

A continuación, se comenta brevemente el contenido de cada etapa y cada técnica. Para una exposición más extensa véanse los trabajos de Botella y Feixas (1998), Crittenden y Ashkar, (2012) o Kelly (1955).

\section{Primera reacción hacia el texto}

La primera etapa es la toma de contacto con el texto, se busca alejar al clínico de aproximaciones categoriales o sintácticas, tanto de tipo lingüístico como psicológico. Para Kelly, el léxico empleado es considerado como un tipo de personalización. Aconseja subrayar las frases pertenecientes a temáticas similares para posteriormente yuxtaponerlas.

\section{Técnicas de análisis}

La segunda etapa consiste en la aplicación de un conjunto de técnicas que pretenden estructurar lo que el clínico está viendo. Observar la secuenciación y transición entre los distintos apartados del texto tiene como objetivo extraer aquellos momentos en los que aparece una discontinuidad, ya sea sobre el tema que se está leyendo, ya sea por el cambio de modo sintáctico dentro de la propia frase. Las discontinuidades, según Kelly (1955), son intentos de equilibrio entre la visión continua -más estructural-del yo y las momentáneas o específicas, y, por tanto, no deben leerse como discontinuidades o rupturas reales.

Respecto a la organización, el objetivo es la búsqueda de las zonas de máxima seguridad, es decir, la exposición de características superficiales de la personalidad que no ponen en juego la identidad global (roles no nucleares) y que no conducen a sentimientos de vulnerabilidad. Estas zonas se exponen, según Kelly, al inicio de cada párrafo, siendo la primera frase del texto la más importante. La siguiente técnica, contraposición hacia el contexto, consiste en reubicar las frases significativas en nuevos contextos 
donde el significado pudiera cambiar drásticamente. La recopilación de términos consiste en encontrar aquellas ideas que se repiten con una frecuencia elevada, se trata de términos sinónimos u homónimos que pueden indicar la presencia de un constructo que no está lo suficientemente elaborado. El cambio de énfasis consistiría en cambiar la entonación de una misma frase sucesivamente, para evitar los posibles sesgos que impidiesen entender lo que el autor del texto nos está queriendo decir. La actualización del argumento consiste en reelaborar el texto bajo el sistema de construcción personal del especialista, esto es, redactar el texto con sus propias palabras. Con esta técnica, Kelly pretendía que el terapeuta se emplazase en el lugar del autor y que sintiese lo mismo que el autor siente.

\section{Análisis de las áreas contextuales}

Para Kelly, las áreas contextuales son las áreas de trasfondo de las que el texto habla, como, por ejemplo, el pasado, la vocación del autor, etc. Estas áreas son las que, desde la perspectiva del sujeto, le hacen diferente al resto. El cliente elabora su propio sistema de construcción personal desde estas áreas. En algunos casos, este trasfondo será muy reducido, y en otros casos, el autor aprovechará para explorar aquellas áreas que tiene menos elaboradas. Estas últimas serán aquellas donde el cliente pueda experimentar cambios con una mayor facilidad y dónde haya mayores posibilidades para el cambio.

\section{Análisis temático}

El análisis temático trata de identificar las relaciones de causa-efecto indicadas en el texto. Estas relaciones pueden encontrarse en frases aisladas o en el propio argumento. Independientemente de la estructura lógica subyacente, para el autor del texto existirá una relación causal conectada psicológicamente. Desde aquí, el terapeuta podrá identificar cómo el cliente enfoca el cambio. Características como la disculpa o la justificación son tareas que Kelly consideraba estrategias para mantener el yo bajo control.

\section{Análisis dimensional}

Es el área de análisis más significativa. En esta etapa el terapeuta presta especial énfasis a las alternativas dico- tómicas (constructos) que el cliente elige para sí mismo. Habrá que identificar aquellos que aparecen y aquellos que están implícitos en el texto. Estos constructos nos dan información sobre la estructura del sistema.

\section{Subsumir los constructos personales del especialista a los del cliente}

La última etapa del análisis consiste en reelaborar el texto integrando el sistema de construcción personal del terapeuta en el del autor. Esto es, se identifican los constructos propios y se incorporan los del cliente, traduciéndolos en términos del terapeuta. Con ello, se pretende elaborar las ramificaciones del sistema del autor del texto para usarlos en futuras exploraciones. Se trata de comprobar cómo el terapeuta puede predecir los términos del sistema del cliente.

\section{Alcance}

Debido a que no se han encontrado trabajos de revisión sistemática o metaanálisis que recopilen los principales hallazgos sobre la autocaracterización, la presente investigación trata de ofrecer una recopilación de los principales trabajos generados al amparo de esta técnica, analizar los trabajos sobre esta y establecer diferencias atendiendo a las instrucciones, a los protocolos de análisis y aquellas áreas en las que se enfocan. Para ello, se lleva a cabo la exploración de los principales trabajos que han empleado esta técnica teniendo en cuenta la tipología del estudio (teórico o empírico), las metodologías empleadas (cualitativa, cuantitativa, mixta), las tipologías de instrucciones y de corrección -clásica o alternativa- (véase Apéndice A). Estas tres características permiten formular comparaciones entre los distintos trabajos consultados. Así mismo, se pretende identificar cuáles son los apartados del análisis que más atención han acaparado y tratar de aducir un motivo que justifique su interés. La finalidad radica en hacer homogéneos los resultados de los artículos revisados tal y como algunos sistemas de clasificación han ofrecido en otras investigaciones (Booth, Papaioannou y Sutton, 2012).

La búsqueda bibliográfica ha estado asociada a la consulta de cinco bases de datos Psycinfo, ScienceDirect, PsicoDoc, WoS y PubPsyc, junto con el uso de otros recursos 
electrónicos fuera del registro de bases de datos automatizadas como revistas electrónicas no indexadas o capítulos de libros que trataban sobre la autocaracterización. Los descriptores empleados han sido self-characterization, self characterization o el término en castellano de autocaracterización.

El artículo se divide en cuatro secciones principales que tienen que ver con los objetivos planteados. En primer lugar, se expondrán los estudios atendiendo a la tipología y a la metodología. En segundo lugar, se tratará de retratar las formas de uso y de corrección de la autocaracterización. En tercer lugar, se repasarán que etapas del análisis han sido más empleadas independientemente de las instrucciones y del formato de análisis. En la última sección, se concluirá con cuáles son las futuras líneas de investigación, su implicación en otras áreas y las propias limitaciones que ofrece la autocaracterización como técnica individual.

\section{Tipologías de estudios}

Los estudios analizados fueron divididos en función de la tipología (teórica o empírica) y, posteriormente, en torno a la metodología aplicada (cualitativa, cuantitativa y mixta). Los estudios teóricos recogen aquellos trabajos publicados que recopilan información sobre la técnica, mientras que los estudios empíricos presentan resultados concretos a través de la aplicación de un procedimiento específico. Respecto a la metodología aplicada, se plantea que los estudios cualitativos agrupan procedimientos y resultados de carácter nominal, o que relacionan contenidos de la autocaracterización con cuestiones de carácter semántico y/o semiótico; los estudios cuantitativos permiten obtener datos concretos que sirven para hacer valoraciones de tipo ordinal o escalar; los estudios mixtos engloban procedimientos y resultados que integran ambos tipos de metodologías, cualitativa y cuantitativa.

\section{Estudios teóricos}

En el grupo cualitativo, los trabajos recopilados mostrabanalgunas indicaciones sobre el procedimiento y presentaban algunas de sus ventajas y limitaciones (Botella y Feixas, 1998; Denicolo, 2003; Hardison y R. A. Neimeyer, 2012; Trucneckova y Viney, 2012). En estos trabajos se destaca la utilidad clínica, el acercamiento crédulo, el formato de las instrucciones y el proceso de análisis, pero ello no quiere decir que no se encuentren contradicciones como las referidas a la deseabilidad social. Para Kelly, el formato de las instrucciones impedía su aparición, mientras que Hardison y R. A. Neimeyer (2012) sostienen lo contrario. Otros trabajos (e.g., Cote, 1995; Trucneckova y Viney, 2012), conciben la técnica como una herramienta que tiene su utilidad en la terapia de rol fijo o en las tareas de consejero escolar, permitiendo una comprensión amplia del sistema de construcción del estudiante a través de subsumir el sistema de construcción personal del consejero al del estudiante.

En el grupo cuantitativo, hay diferentes propuestas recogidas en los documentos de Blowers y O'Connor (1995), B. Green (2004), Jackson (1988), Viney y Caputi (2012). El trabajo de Blowers y O'Connor (1995) destaca la utilidad para explorar constructos subyacentes, los intentos de llevar a cabo un análisis cuantitativo de la técnica y como herramienta alternativa a la rejilla. Los artículos de B. Green (2004) y Viney y Caputi (2012) se enmarcan dentro del enfoque de análisis de contenido. En el artículo de B. Green (2004) se incide en los trabajos de McAdams (1994) y de Jackson (1988) en relación a la cuantificación de la autocaracterización. Además, se indica que los constructos personales no equivalen necesariamente a términos concretos y que el polo de un constructo no es una entidad suficiente para establecer algún tipo de inferencia (B. Green, 2004). Por su parte, Viney y Caputi (2012) han recopilado información a través de la elaboración de distintas escalas para medir conceptos como, por ejemplo, el tono emocional y la ansiedad cognitiva dentro de instrumentos como la autocaracterización. Así, estas escalas han permitido establecer disimilitudes en cuanto al género, la edad o la salud. Jackson (1988) asoció una medida cuantitativa dentro de la autocaracterización a un conjunto de corolarios. De esta manera, propuso distintas medidas que cuantificaban la autoestima, el tipo de declaraciones empleadas, la visión que la persona tiene sobre los demás, las relaciones de causa-efecto psicológicas o el grado de insight alcanzado.

Atendiendo a una metodología mixta, el capítulo escrito por Crittenden y Ashkar (2012) describe dos metodologías, clásica y otra fundamentada en la multiplicidad 
de yoes de Mair (1977), para emplear la autocaracterización.

Una de las principales aportaciones de los trabajos de corte teórico es la descripción de diferentes estilos de corrección de la autocaracterización, denotando que no existe un único formato de análisis válido. Aquellos elementos comunes que aparecen destacados en estos trabajos como señales univocas serían el acercamiento crédulo y la utilidad clínica. Respecto al acercamiento crédulo, se guía al terapeuta para aproximarse al texto como si de una verdad narrativa se tratase. Es decir, se pretende ver el mundo a través de los propios ojos del cliente sin que sea necesario cuestionar la realidad objetiva del relato. En cuanto a la utilidad clínica, tiene que ver con la flexibilidad con la que se pueden modificar las instrucciones en función de las necesidades del clínico. De la misma manera, se pone de relieve el problema de la deseabilidad social que pudiera estar vinculada a las propias instrucciones de la técnica.

\section{Estudios empíricos}

Los estudios de corte cualitativo pueden clasificarse según el número de casos descritos en el estudio (uno o más de uno). Dentro de los estudios de caso único, se encuentran los trabajos de S. J. Bell y R. C. Bell (2008), Feixas y Gallinat, (1989), B. Green (2016), R. A. Neimeyer et al. (2003), Reed et al. (2014), y Winter y Tschudi (2015). En el estudio de S. J. Bell y R. C. Bell (2008) se utilizó una técnica proyectiva de clave pictórica para elaborar una autocaracterización en el análisis de un niño de siete años. Los autores concluyen que la interpretación del proceso de construcción depende de la habilidad del terapeuta, tanto en el caso de uso de la técnica de autocaracterización, como en el dibujo. El trabajo de Feixas y Gallinat (1989) se enmarca en la terapia del rol fijo, la autocaracterización es presentada como una herramienta que sirve para conocer el sistema de construcción personal del individuo, dónde se sitúa el yo respecto a las dimensiones que estructuran el mundo de la persona y ver qué tipo de relación/rol establece el sujeto con el mundo que le rodea. En el estudio de R. A. Neimeyer et al. (2003), se emplearon la autocaracterización y la terapia del rol fijo con una mujer que acudía a la unidad de psicología clínica en la universidad. Se aplica la técnica del rol fijo a través de un análisis estructurado en 14 fases. La terapia sirvió para que la cliente dejase de usar un patrón rígido en la anticipación de la experiencia. Los artículos de Reed et al. (2014), Winter y Tschudi (2015) y Green (2016) analizan tres casos clínicos a través de sendas obras literarias. En su conjunto, exploraron de la evitación del sentimiento de culpa del comandante Hess (Reed et al., 2014); la ausencia de constructos que clasifican personas en la figura de Breivik (Winter y Tschudi, 2015); la evolución de las emociones de aislamiento e inferioridad hacia sentimientos de venganza y castigo hacia los demás en un asesino en serie (B. Green, 2016).

En el segundo grupo, se encuentran estudios que aplican la autocaracterización en contextos diferentes al análisis de caso único y muestran resultados de varios individuos (e.g., Androutsopoulou, 2001; Ashkar, 2015; Faccio Cipolleta, Romaioli y Ruiba, 2011; D. Green y KirbyTurner, 1990; Hopkins, 2012; Lovenfosse y Viney, 1999; Trucneckova y Viney, 2007; Veronese, Procaccia, Ruggiero, Sassaroli y Castiglioni, 2015). Concretamente, se diferencian tres modelos de estudios: (a) Aquellos que emplean la autocaracterización como variable independiente (e.g., Androutsopoulou, 2001), (b) estudios que buscan explorar la asociación entre variables (e.g., Ashkar, 2015; Veronese et al., 2015); y (c) los que se centran en la aplicación de un procedimiento sobre el texto de autocaracterización cuyo resultado es la variable dependiente del estudio (e.g., Faccio et al., 2001; D. Green y Kirby-Turner, 1990; Hopkins, 2012; Lovenfosse y Viney, 1999; Truneckova y Viney, 2007). En el estudio de Androutsopoulou (2001), se aplican tres tipos diferentes de instrucciones: una versión similar al original, otra del "Yo-ideal" dentro de cinco años y otra destinada a caracterizar a la familia. Androutsopoulou (2001) concluye que la metáfora del teatro es útil en la caracterización de la familia, ya que permite y facilita la labor terapéutica, acercando categorizaciones entre sí, lo que hace homogéneo a un grupo familiar. El trabajo de Askar (2015) pretendía observar la asociación entre el contenido temático de los constructos y su asociación con el bienestar emocional y psicológico. En el estudio se solicitaron hasta un total de seis autocaracterizaciones que representaron diferentes roles de los participantes (como estudiante/como pareja, etc...). Sus resultados vincularon el contenido temático de los constructos con la variable bienestar, siendo 
el contenido temático equivalente independientemente del rol solicitado en la autocaracterización. En el trabajo de Faccio et al. (2011), se trata de identificar, mediante la autodescripción, los constructos asociados en personas diagnosticadas de bulimia en dos situaciones hipotéticas, con control y sin control, y en dos momentos distintos, al inicio del tratamiento y al final (más allá del problema). Los resultados indicaron que la autocaracterización aplicada a la situación fin del tratamiento permite encontrar las direcciones de cambio y facilita la posibilidad de generar historias alternativas. En el estudio de D. Green y Kirby-Turner (1990) se exploran los esquemas que los terapeutas de diferentes escuelas manifiestan cuando están comenzando a trabajar como terapeutas familiares. La metodología propuesta para el estudio pretende capturar el cambio en las construcciones de los terapeutas e implicaba extraer los constructos completos de las autocaracterizaciones y agruparlos en una rejilla textual. Los autores lograron establecer diferencias de carácter cualitativo, ya que en el nivel individual se encontraron cambios en los postulados que los terapeutas mantenían antes de comenzar como terapeutas familiares. En el documento de Hopkins (2012) se estudian los constructos asociados con la sensibilidad, la naturaleza social, la mente cerrada, la organización y la energía o fortaleza en personas con agorafobia y sin agorafobia, usando el sistema de categorías de Landfiel (1971). El grado de acuerdo entre evaluadores fue modesto (Kappa $=$ 0.59). Los resultados indicaron que las personas con agorafobia se describen como altamente sensibles, en comparación con el grupo sin agorafobia. El artículo de Lovenfosse y Viney (1999) se centra en identificar los significados personales de doce madres de niños con necesidades especiales y explorar el posible beneficio obtenido al participar en un grupo de trabajo. Para generar los significados personales, se les aplicó la autocaracterización y se les instó a identificar sus fortalezas y limitaciones. Se concluyó que se describían como personas cariñosas que cuidaban más de los suyos que de sí mismas. El trabajo de Trucneckova y Viney (2007) se centró en explorar el cambio en intervención en adolescentes problemáticos a través de la exploración de constructos hallados en la autocaracterización. Una vez eran identificados se clasificaban atendiendo al contenido temático y a los niveles de abstracción de los constructos. Mediante estas dos categorías los autores, a través del tratamiento, informaron de cambios se dirigían hacia unos constructos más interpersonales y con un mayor nivel de abstracción. En el último trabajo de este grupo (Veronese et al., 2015), se utilizó la autocaracterización para la identificación de estructuras narrativas subyacentes en los intentos narcisistas para proteger el yo. Mediante una clasificación de fragmentos de contenido de los textos en tres dimensiones, amenazas y defensas de la autoestima y respuestas de relajación, se vincularon dimensiones relacionales con los dominios de amenazas y de defensa.

En el grupo de artículos cuantitativos encontramos cuatro artículos (Hardison y R. A. Neimeyer, 2007; Procaccia, Veronese y Castiglioni, 2014; Vall y Botella, 2014; Winter, Gournay, Metcalfe y Rossotti, 2006). Hardison y R. A. Neimeyer (2007) estudiaron el grado de convergencia entre tres técnicas de la TCP, la autocaracterización, la técnica de rejilla y la técnica de escalamiento, atendiendo a la diferenciación y complejidad de los constructos empleados, la autoestima y el contenido temático. Los resultados indicaron que no existía relación entre ninguna de ellas ni para la complejidad cognitiva, ni para la autoestima. Sin embargo, en todos los casos, las áreas de contenido temático (medidas a través del sistema de Feixas et al., 2002), que se presentaron en mayor proporción fueron la emocional, la personal y la relacional. En el estudio de Procaccia et al. (2014) se exploran autocaracterizaciones infantiles usando un protocolo de análisis de tipo cuantitativo. Usan una muestra de 103 niños a la que se aplica un test de ansiedad por separación y una autocaracterización. Los resultados de este procedimiento indicaron que había diferencias en las autocaracterizaciones en cuanto a edad, género y estilo de apego. El estudio de Vall y Botella (2014) se basa en el estudio de las macroestructuras narrativas, es decir, en el estudio de la organización de la narrativa. Se quiso comprobar si había diferencias estadísticamente significativas en torno a las macroestructuras narrativas cuando se presentaba una puntuación alta en ansiedad o en depresión en una muestra de 29 individuos. El procedimiento de análisis de autocaracterización empleado fue la rejilla de evaluación narrativa (Botella y Gamiz, 2011), que se divide en siete etapas. La amplia mayoría de las etapas resultaron útiles para diferenciar los dos tipos de psicopatología. Winter et al. (2006) emplean la técnica como una herramienta intersesiones para la investigación en personas con agorafobia en el marco de una interven- 
ción psicoterapéutica sobre constructos personales. Los autores consiguieron unos resultados satisfactorios a favor de la intervención en comparación a la lista de espera.

El grupo de metodología mixta agrupa los artículos de Buckley-Walker, Crowe y Caputi (2013), Hamad y Lee (2013), Veronese, Procaccia y Castiglioni (2012) y Veronese Procaccia, Romaioli, Barola y Castiglioni (2013). El primer estudio presenta un método para analizar constructos de familiares de personas con enfermedad mental a través del sistema de clasificación de constructos de Feixas, Monferrer, Geldschläger y Ruiz (2002), en una muestra de 18 personas con dos estilos de instrucciones distintas de autocaracterización, una centrada en el "YoAhora" y otra en el "Yo-Ideal". Los resultados fueron convertidos en una rejilla y mostraron que las áreas menos representadas fueron la relacional, la intelectual y la de valores e intereses. El segundo estudio se valió de la aplicación de la técnica de rejilla y la autocaracterización para explorar la experiencia vivida por estudiantes internacionales en los países de acogida. Los autores concluyen que autocaracterización y rejilla ofrecen constructos diferentes. La autocaracterización ahonda en roles ejercidos (país de origen-país de acogida). Los otros dos artículos del grupo mixto se centran en la relación entre familias semánticas, estructuras de apego y diagnósticos en casos de fobia a volar. Los autores parten de la idea de que la manifestación de problemas fóbicos o de tipo ansioso tiene su origen en las temáticas conversacionales familiares referidas a los constructos "Libertad/Dependencia" y "Miedo/Valor". En ambos estudios, los términos del grupo con fobia se asociaron a la familia semántica "Libertad/dependencia" y "Distante/Cercano".

Los trabajos empíricos ponen de manifiesto la flexibilidad de la propia técnica dando énfasis a modos de instrucción y de corrección alternativos. Al igual que hacen los trabajos de carácter teórico, también se ha destacado la utilidad clínica de la autocaracterización. Su uso ha ido asociado con la exploración de las emociones, direcciones de cambio, sistema de construcción personal (contenido temático), caracterización de los constructos personales en grupos poblacionales específicos, terapia de rol fijo o conversión en rejilla. No obstante, se han encontrado ciertas dificultades en homogeneizar los resultados atendiendo a la disparidad de estrategias de análisis y formato de ins- trucciones. Se ha observado que sí hay cierto consenso en lo que se quiere explorar pero no en el cómo hacerlo.

\section{Protocolos de instrucciones y de corrección de la autocaracterización}

En cuanto a las instrucciones, se han encontrado aquellos que no hacen mención a estas (Cote, 1995; Blowers y O'Connor, 1995; B. Green, 2004; Trucneckova y Viney, 2012; Viney y Caputi, 2012) y otros estudios que tratan con formatos de instrucción clásica o alternativa (Androutsopoulou, 2001; Ashkar, 2015; Buckley-Walker et al., 2013; Crittenden y Ashkar, 2012; Winter et al., 2006). En su conjunto, en estos estudios el interés parte de contemplar las divergencias entre las diferentes versiones del yo, confrontando la visión del yo en un momento presente, respecto a una versión ideal, una construcción familiar o una versión sin el problema. Por otro lado, un número relevante de estudios han enunciado exclusivamente las instrucciones clásicas (Denicolo, 2003; Botella y Feixas, 1998; Feixas y Gallinat, 1989; Hamad y Lee, 2013; Hardison y R. A. Neimeyer, 2007, 2012; Lovenfosse y Viney, 1999; R. A. Neimeyer et al., 2003; Trucneckova y Viney, 2007; Vall y Botella, 2014; Veronese et al., 2012; Veronese et al., 2013; Veronese et al., 2015), mientras que otros han hecho uso de formatos de instrucción alternativos (S. J. Bell y R. C. Bell, 2008; Faccio et al., 2011; D. Green y Kirby-Turner, 1990; B. Green, 2016; Hopkins, 2012; Jackson, 1988; Procaccia et al., 2014; Reed et al., 2014; Winter y Tschudi, 2015). Los trabajos de S. J. Bell y R. C. Bell (2008) y Jackson (1988) presentan instrucciones específicas. En el primer trabajo, se empleó la siguiente instrucción: «Voy a pedirte que hagas algunos dibujos. Al dibujar puedes hablar en voz alta sobre tus dibujos, si quieres. Una vez hayas acabado tu dibujo, te preguntaré sobre lo que has dibujado. Me gustaría que pensases que en estas hojas de papel como un lugar donde estar, como a ti te gustase. Es una parte de tu mundo, un lugar donde estar mientras dibujas. Si dibujas cualquier cosa que te guste, estaré feliz. Es tu espacio para dibujar lo que te gustaría» (S. J. Bell y R. C. Bell, 2008, traducido por los autores). En el segundo trabajo, se utilizó la siguiente instrucción: "Cuéntame qué clase de chico o chica es Sally. Si quieres podré ser tu secretario/a y apuntar lo que digas. Háblame sobre ti mismo/a como si 
fueras a ser descrito por un amigo imaginario que te conoce y te quiere y te comprende muy bien. Esta persona debe ser capaz de decir cuál es tu carácter y todo sobre ti. Quizás podrías comenzar con Sally es... y decir algo importante sobre ti mismo/a. Intenta completar esta página» (Jackson, 1988, traducido por los autores). Los trabajos de B. Green (2016), Reed et al., (2014) y Winter y Tschudi, (2015) han empleado textos escritos como si fuesen autocaracterizaciones mientras que, el resto han realizado modificaciones en las instrucciones atendiendo al objeto de la exploración (cualidades específicas, profesión, problemas, superación de problemas).
En cuanto a los formatos de corrección, se tuvo en cuenta los trabajos que no hacían mención explícita al procedimiento de análisis (Androutsopoulou, 2001; Blowers y O'Connor, 1995; Cote, 1995; Denicolo, 2003; Feixas y Gallinat, 1989; Trucneckova y Viney, 2012; Winter et al., 2006), aquellos que utilizan exclusivamente el protocolo básico (S. J. Bell y R. C. Bell, 2008; Feixas y Botella, 1998); aquellos que presentan una versión alternativa o que se focalizan en un aspecto concreto del protocolo (Ashkar, 2015; Buckley-Walker et al., 2013; Faccio et al., 2011; B. Green, 2004, 2016; D. Green y Kirby-Turner, 1990; Hamad y Lee, 2013; Hardison y R. A. Neimeyer, 2007; Hopkins, 2012; Jackson, 1988; Lovenfosse y Viney,

Tabla 1

\section{Protocolos de corrección de autocaracterización}

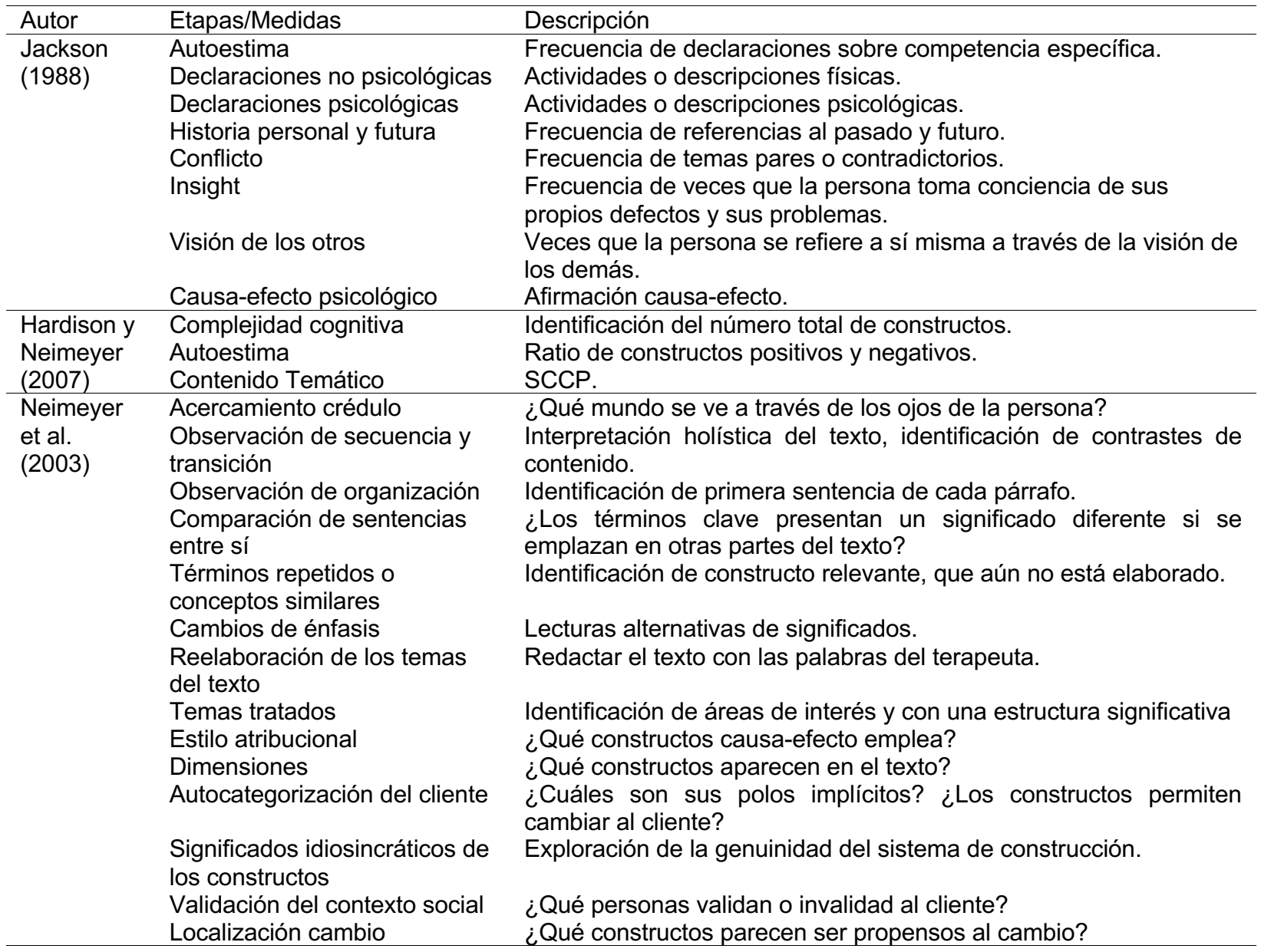


Tabla 1 (continuación)

Protocolos de corrección de autocaracterización

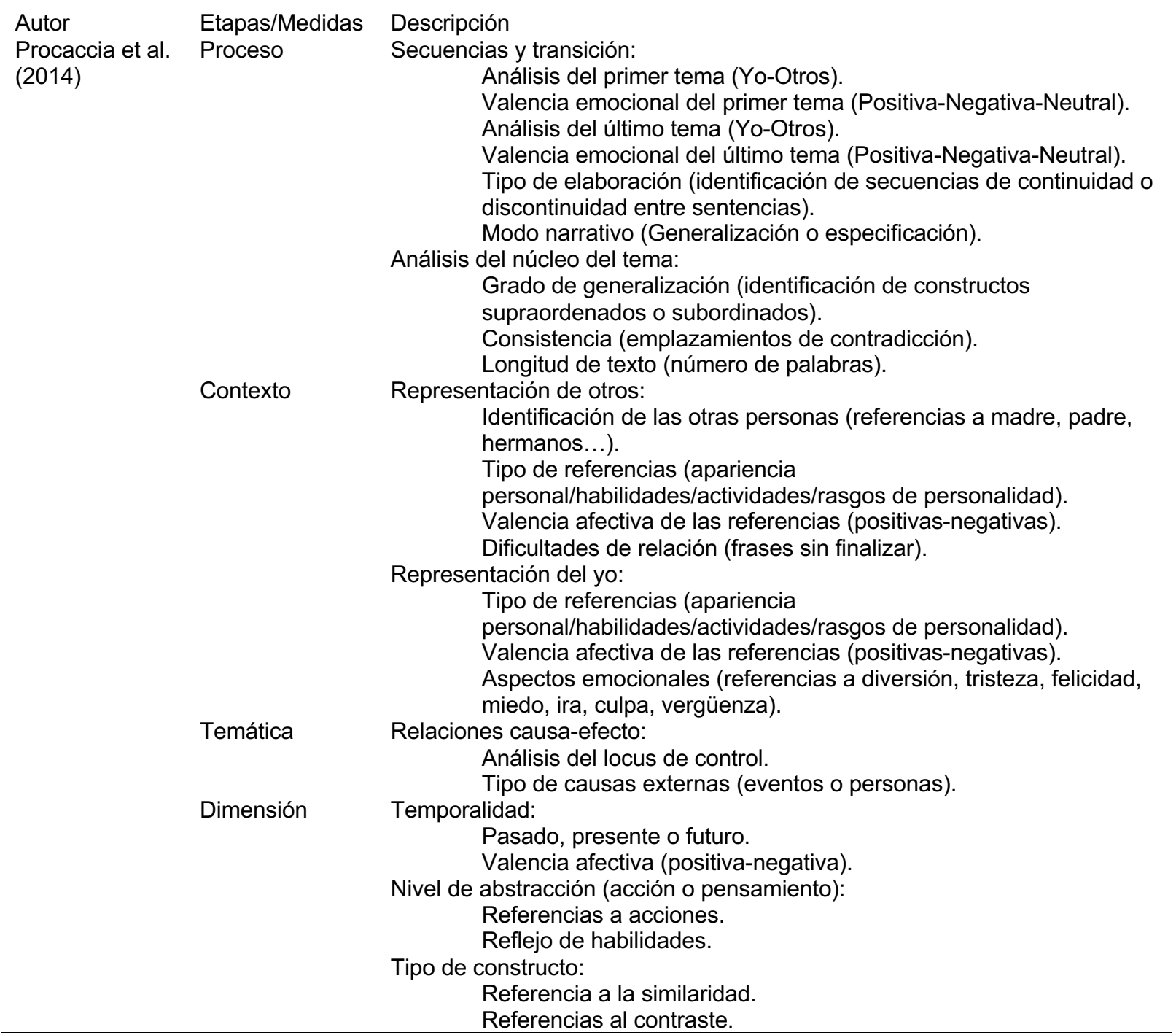


Tabla 1 (continuación)

Protocolos de corrección de autocaracterización

\begin{tabular}{|c|c|c|}
\hline Autor & Etapas/Medidas & Descripción \\
\hline \multirow[t]{6}{*}{$\begin{array}{l}\text { Vall y Botella } \\
(2014)\end{array}$} & $\begin{array}{l}\text { Estructura y coherencia } \\
\text { narrativa }\end{array}$ & $\begin{array}{l}\text { Orientación general (quién aparece en el relato, cuándo sucede y } \\
\text { dónde tiene lugar). } \\
\text { Secuencia estructural (qué se narra). } \\
\text { Evaluación del cometido (porqué se narra). } \\
\text { Integración (grado en que el argumento está interconectado). }\end{array}$ \\
\hline & $\begin{array}{l}\text { Contenido narrativo y } \\
\text { pluralidad }\end{array}$ & $\begin{array}{l}\text { Variedad temática. } \\
\text { Variedad de eventos. } \\
\text { Variedad de escenarios. } \\
\text { Variedad de personajes. }\end{array}$ \\
\hline & $\begin{array}{l}\text { Proceso y complejidad } \\
\text { narrativa }\end{array}$ & $\begin{array}{l}\text { Objetivación (detalles sensoriales). } \\
\text { Subjetivación emocional (cantidad de detalles emocionales } \\
\text { asociados a eventos). } \\
\text { Subjetivación cognitiva (diferentes términos internos para describir } \\
\text { pensamientos). } \\
\text { Uso de metáforas. }\end{array}$ \\
\hline & Inteligibilidad narrativa & $\begin{array}{l}\text { Grado de claridad de la meta narrativa. } \\
\text { Conflicto entre las diferentes metas. } \\
\text { Plausibilidad de la última meta. } \\
\text { Eventos relevantes para conseguir la meta. } \\
\text { Eventos implicados en una secuencia. } \\
\text { Estabilidad de los personajes. } \\
\text { Vínculos causales. }\end{array}$ \\
\hline & $\begin{array}{l}\text { Otras dimensiones } \\
\text { relevantes }\end{array}$ & $\begin{array}{l}\text { Secuencia de contaminación (Comienzo progresivo, final } \\
\text { regresivo). } \\
\text { Secuencia de redención (comienzo regresivo, final progresivo). }\end{array}$ \\
\hline & Posición narrativa del yo & $\begin{array}{l}\text { Protagonista indefenso. } \\
\text { Protagonista sujeto a las circunstancias. } \\
\text { Protagonista con control intermedio de sus acciones. } \\
\text { Protagonista proactivo. } \\
\text { Protagonista luchador. }\end{array}$ \\
\hline
\end{tabular}

1999; R. A. Neimeyer et al., 2003; Procaccia et al., 2014; Reed et al., 2014; Trucneckova y Viney, 2007; Vall y Botella, 2014; Veronese et al., 2012; Veronese et al., 2013; Veronese et al., 2015; Viney y Caputi, 2012; Winter y Tschudi, 2015) y aquellos que presentan conjuntamente las dos versiones (Crittenden y Ashkar, 2012; Hardison y R. A. Neimeyer, 2012). Los artículos que no han tratado ningún procedimiento han centrado su atención en la descripción teórica de la técnica o su vinculación con el rol fijo. Respecto a los trabajos que han establecido sistemas de análisis alternativos, se han centrado en clasificar el contenido temático de los constructos personales mediante sistemas de clasificación generados ad-hoc o el uso de instrumentos como el SCCP de Feixas et al. (2002) o el sistema de clasificación de Landfield
(1971). Mención aparte sería el trabajo Viney y Caputi (2012) que es presentado como un conjunto de escalas para valorar el contenido de la autocaracterización. Estas escalas: origen, empeño, afecto, gregarismo, madurez psicosocial y ansiedad valoran el grado de éxito, fracaso, sentimientos positivos, las relaciones sociales, fases del desarrollo a lo largo de la vida y el concepto de ansiedad propuesto por Kelly, respectivamente. Los trabajos de Jackson (1988), Hardison y R. A. Neimeyer (2007), Procaccia et al. (2014) y Vall y Botella (2014) (Tabla 1) han presentado protocolos de corrección diferentes de aquellas fórmulas que analizan el contenido temático de los constructos. Algunas de estas propuestas se acercan bastante al procedimiento clásico (R. A. Neimeyer et al., 2003), mientras que otras están más fundamentadas en 
otros enfoques teóricos, más propios de terapias narrativas (Vall y Botella, 2014).

De los dos trabajos que exponen estrategias clásicas y alternativas, el trabajo de Crittenden y Ashkar (2012), que ahonda más en la metodología alternativa, describe un procedimiento fundamentado en la teoría de los múltiples yoes de Mair (1977). El análisis consiste en elaborar diferentes autocaracterizaciones asociadas a los distintos roles, extraer las polaridades de constructos y utilizar los roles a modo de elementos para confeccionar una rejilla. Esta metodología tendría ciertas similitudes con la rejilla textual propuesta por Feixas y Villegas (1991).

En resumen, se puede argumentar que las instrucciones pueden ser alteradas en función del objetivo que se tenga. El acercamiento crédulo, el uso de tercera persona y la intimidad del texto son aspectos fundamentales que parecen estar presentes en aquellos trabajos que especifican las instrucciones dadas. En cuanto a las diferentes estrategias de corrección, hay un cierto interés por tratar de extraer datos concretos que sean útiles para la labor terapéutica e investigadora. También se observa que, pese a ser una técnica creada al margen de procedimientos psicométricos, se han dado algunos pasos en este aspecto, siendo el trabajo de Jackson (1988) un ejemplo representativo en este sentido. Pese a la aparente divergencia de intereses y metodologías empleadas hay ciertos aspectos del análisis de la autocaracterización que se han repetido, ya sea por la influencia del procedimiento original o por la utilidad del dato que se obtiene de su corrección.

\section{Áreas de análisis que han despertado más interés}

Cuando se atiende al procedimiento de análisis de una autocaracterización hay ciertos aspectos que tienen que ver con la actitud que el analista debe sostener respecto al texto. Estas actitudes se recopilan en las etapas: "Primera reacción hacia el texto", "Cambio de énfasis y Actualización de argumento" $y$ "Subsumir los constructos personales del especialista a los del cliente". En su conjunto permiten diferenciar esta técnica hermenéutica constructivista de otras. Aunque no son descritas frecuentemente, quizás dicha ausencia sea debida a una presuposición sobre dichas actitudes, ya que al ser trabajos fundamentalmente enmarcados en una postura epistemológica constructivista puede darse por supuesto este hecho. Desde otro punto de vista, el hecho de que dichas etapas no ofrezcan resultados concretos puede ser otro motivo.

Otras áreas como la exploración de la primera sentencia del texto, la búsqueda de sentencias causa-efecto, discontinuidades temáticas, términos repetidos o la identificación de los temas que aparecen, son aspectos que sí han capturado interés de lo que se desprende de las diferentes estrategias de análisis. Esto puede verse debido a que dichas áreas capturan lo que interesa al autor del texto y ofrece un alto valor de sistematicidad en la obtención de datos. De tal manera que, mediante unos pocos procedimientos en el análisis de texto, se pueden encontrar qué términos son los que más se repiten o dónde hay una ruptura en el discurso narrativo del autor del texto, etc. Estos datos, en esencia, capturan el proceso psicológico de la persona (G. J. Neimeyer, 1996). Los contenidos de estas etapas facilitan la interpretación del texto en torno a unos valores concretos, ya que permite identificar el proceso de diferenciación y subjetivación entre un individuo y otro.

Teniendo en cuenta esto, junto con el desarrollo de la propia TCP muy vinculada al estudio de la técnica de rejilla, se comprende que el área que más interés ha despertado es la exploración del constructo personal, esto es "Análisis Dimensional". La amplia mayoría de los trabajos destacados han centrados sus esfuerzos en la identificación y clasificación temática de los constructos personales. Los trabajos llevados a cabo por Veronese et al. (2012) en grupos clínicos dan constancia de ello. Uno de los principales motivos por los que genera tanto interés es la posibilidad de llevar a cabo clasificaciones, ya que su estudio facilita la identificación de la estructura de sistema de construcción personal y posibilita la comparación con otras técnicas de evaluación constructivista. Permite la agrupación de individuos y su posterior comparación.

En conclusión, se observa que pese a proponer diversas estrategias de análisis de autocaracterización, ciertos aspectos de la exploración clásica se han mantenido vigentes. 


\section{Conclusiones}

La presente revisión ha servido para recopilar y exponer las áreas de interés y los resultados obtenidos atendiendo a la naturaleza y a la metodología de los estudios. En este sentido, varios aspectos han sido destacados a lo largo de la revisión. Los trabajos más recientes, aquellos publicados en la última década, están asociados a una tipología empírica y metodología cualitativa. Concretamente, los autores se han interesado más por diseños que tienen en cuenta muestras mayores de $n=1$. Los estudios cualitativos han recopilado los ámbitos de aplicación de la técnica, definidos por la intervención clínica (e.g., rol fijo, estudio del cambio asociado al proceso de tratamiento, exploración de emociones), la descripción de construcciones subjetivas asociadas a entidades psicopatológicas, la relación entre variables psicológicas o la categorización temática de las construcciones de los textos. Los estudios cuantitativos han reflejado la preocupación por la extracción de datos a través de escalas y la comparación de resultados con otras técnicas. Los estudios mixtos han recogido trabajos centrados en la exploración de constructos propios de psicopatologías concretas, la comparación de resultados entre técnicas enmarcadas dentro de la TCP o la exploración de roles.

Otro de los objetivos era estudiar tanto las tipologías de instrucción y de procedimiento de análisis y las etapas del mismo que más interés han despertado. En las investigaciones recopiladas, aquellas donde se especificaban instrucciones se hacía alusión a dos aspectos: el acercamiento crédulo y la flexibilidad de las instrucciones. El énfasis del acercamiento crédulo tiene que ver con las actitudes que se deben seguir a la hora de corregir una autocaracterización y con el concepto de verdad narrativa propuesto por Spence (1982), basado en la idea de versosimilitud, citado por Botella y Feixas (1998), que indica que una historia es buena o válida en la medida en que la narración es conveniente y coherente. La autocaracterización parece deber su utilidad clínica a la flexibilidad y a la amplitud de las instrucciones dadas. Otras técnicas narrativas, como las historias de vida (McAdams, 1993), precisan de una formulación específica de instrucciones para la descripción concreta de sucesos, de un marco que haga de contexto para la definición personaje, que contemple diferentes momentos temporales (Chan, Ng, Tien, Man y Thayala, 2013;
Dunn, 2017). La autocaracterización no precisa de ese contexto, propone la definición de un personaje desde la visión de otra persona. Así se otorga al autor la libertad de proponer desde una simple descripción hasta una sucesión de hechos que sirven como marco para explicar quién es esa persona. La flexibilidad de las instrucciones tendría que ver con el tipo de dato concreto que quiere obtener el investigador. Respecto a las estrategias de corrección, algunos estudios han optado por explorar versiones alternativas del protocolo de corrección clásico. En este sentido, pese a los esfuerzos llevados a cabo por los distintos autores, ninguna de las propuestas ha tenido mayor repercusión a nivel empírico que las investigaciones realizadas por los autores que han diseñado la propuesta alternativa. Es decir, la generación de nuevas propuestas, no han servido para generar un cuerpo teórico y empírico mucho más rico alrededor de esta técnica. Sin embargo, un aspecto central que se repite en todos los trabajos, y que tiene que ver con el objetivo de explorar cuál es el apartado que más atención ha captado, es la exploración de los constructos personales. Este aspecto se ha visto como el principal elemento a estudiar, con independencia de lo que el investigador solicite y lo que el autor decida narrar. Y es que, el estudio de los constructos personales, cómo la persona a través de su discurso expone la manera en la que captura la esencia de las cosas, no deja de ser uno de los aspectos clave de la TCP. No obstante, a diferencia de otros autores interesados en las narrativas personales (e.g., Bruner 1997, 2003; Dunn, 2017; Gergen, 1996), la autocaracterización se focaliza en el estudio de la elección que hace la persona, del constructo que elige para sí y no tanto en la historia que cuenta, la historia en la que se crea el personaje, o la capacidad que tiene parar narrar la historia.

Se han identificado ciertos problemas que tienen que ver con la deseabilidad social de la técnica, la homogenización de los datos atendiendo al procedimiento de análisis. Tal y como fueron formuladas las instrucciones, la deseabilidad social existirá en la medida que se respeten las instrucciones. Esto es debido a que se pretende una visión positiva de la persona alejada de sesgos negativos sobre la identidad que puedan relacionarse con distintas psicopatologías. Por otro lado, la ausencia de un procedimiento unificador en el análisis de la autocaracterización más allá del que propuso Kelly, deja un vacío en torno a la homogenización de los datos. Y es que en el ámbito psi- 
coterapéutico hay un interés en generar sistemas de codificación de narrativas personales que permitan detectar cambios y estructuras discursivas subyacentes (Di Fabio y Bernaud, 2018). Por tanto, puede que la ausencia de un sistema homogéneo esté lastrando su mayor difusión respecto a otras técnicas como la rejilla o las historias de vida. Si no existe la posibilidad de comparar datos y replicarlos entre estudios, ¿cómo pueden ser de robustas las conclusiones obtenidas?

Futuras áreas de investigación, la necesidad de un procedimiento sistematizado para la obtención de los datos. Atendiendo a esta problemática y contemplando aquellas etapas que más utilidad empírica han manifestado, se podría vislumbrar la utilidad de un procedimiento de análisis que sea representativo del modelo clásico e integre otras metodologías. La difusión de la autocaracterización tiene que ver con cómo es de adaptable a diferentes escenarios tanto terapéuticos como científicos. Por ello, un modelo de análisis que fuese útil debería aunar aquellas etapas esenciales que caracterizan a la técnica. A modo de ejemplo, dicho sistema de análisis debería contemplar el estudio de los constructos personales, la exploración del contexto del texto a través de la identificación de los distintos temas, el estudio del proceso del discurso narrativo de la persona a través de la identificación y clasificación de las sentencias causa-efecto, la exploración de las discontinuidades entre los distintos temas del texto y el estudio del estilo atribucional. De la misma forma, la exploración de los distintos roles y personajes que aparecen en el texto serían un aspecto muy relevante para su análisis. La sistematización del análisis de la autocaracterización puede subsanar todas las deficiencias metodológicas encontradas en los diferentes trabajos, facilitando adquirir un estatus de técnica relevante dentro del marco psicoterapéutico más allá del marco conceptual y epistemológico donde ha sido concebida.

Por otro lado, la sistematización del análisis permitiría la posibilidad de aplicar software al estudio de la autocaracterización. En algunos trabajos, se ha contemplado el uso de diferentes paquetes informáticos para el análisis, ya sea el VOSPEC, CASPM o Atlas TI. Sin embargo, su uso no ha sido más extendido presumiblemente por la dificultad que entraña establecer un análisis más cercano a la cuantificación cuando se trata de textos narrativos o por la escasa sistematización del protocolo de análisis.

\section{Limitaciones de la investigación}

El presente estudio adolece de las limitaciones propias de una investigación de revisión narrativa. Sin embargo, se destaca la imposibilidad de comparar las conclusiones obtenidas, debido a que no han sido hallados estudios que hayan llevado a cabo una revisión del estado actual de la autocaracterización.

\section{Utilidad de la técnica como herramienta de análisis}

En resumen, el hecho de que no se haya visto al mismo nivel que la rejilla u otras técnicas narrativas y que haya sido tratada tradicionalmente como una herramienta complementaria, puede tener que ver con el objeto de análisis de la autocaracterización. Si no se unifica qué se desea medir, identificar o cuantificar es complicado generalizar su uso. Ya se ha comentado que la autocaracterización como técnica permite monitorizar el cambio terapéutico, permite la identificación de la estructura, rasgos y características del sistema de construcción personal, puede ser adaptada a diferentes grupos poblacionales en función del interés del clínico, fomenta la alianza terapéutica, puede ser aplicada grupalmente, facilita la exploración de los diferentes roles individuales, etc. La potencialidad de la autocaraterización es muy amplia y un protocolo de análisis que sistematice la obtención de datos y la significación de estos puede permitir que la autocaracterización se erija como una técnica con suficiente identidad para ser aplicada a diversos contextos clínicos y donde se sostengan diferentes posturas epistemológicas. 


\section{Referencias $^{1}$}

*Androutsopoulou, A. (2001). The Self-characterization as a Narrative Tool: Applications in Therapy with Individuals and Families. Family Process, 40(1), 79-41. https://doi.org/10.1111/j.1545-5300.2001. 401010079

*Ashkar, C. (2015). Finding our many selves: A personal construct psychology approach to positive mental health (Tesis de Máster), Universidad de Wollongong). Wollongong, Australia. Recuperado de

http://ro.uow.edu.au/cgi/viewcontent.cgi?article $=5$ $717 \&$ context $=$ theses

Beach, R. (1977). Writing about ourselves and others (Informe $\mathrm{N}^{\mathrm{o}}$. 400-75-0029). Recuperado de https://eric.ed.gov/?id=ED141831

*Bell, S. J. y Bell, R. C. (2008). An Illustration of Self Characterization in a Child's Drawing: The Importance of Process. Personal Construct Theory \& Practice, 5, 1-9.

Bruner, J. (1997). La educación, puerta de la cultura [The culture of Education]. Madrid, España: Visor Dis.

Bruner, J. (2003). La fábrica de historias: derecho, literatura, vida [Taking Stories: Law, Literature, Life]. México, México: Fondo de Cultura Económica.

*Blowers, G. H. y O’Connor, K. P. (1995). Construing Contexts: Problems and Prospects of George Kelly's Personal Construct Psychology. British Journal of Clinical Psychology, 34(1), 1-16.

Booth, A., Papaioannou, D. y Sutton, A. (2012). Systematic Approaches to a Successful Literature Review. Londres, Reino Unido: Sage.

\footnotetext{
${ }^{1}$ Las referencias marcadas con un asterisco señalan los trabajos incluidos en la revisión.
}

*Botella, L. y Feixas, G. (1998). La exploración del sistema de construcción del cliente [The Exploration of Personal Construct System]. En L. Botella y G. Feixas (Eds.), Teoría de los constructos personales: aplicaciones a la práctica psicológica [Personal Construct Theory: Applications to Psychology Practice] (pp. 64-95). Barcelona, España: Laertes.

Botella, L. y Gamiz, M. (2012). Narrative Assessment in Psychotherapy: A constructivist Approach. En P. Caputi, L. L. Viney, B. M. Walker y N. Crittenden (Eds.), Personal Construct Methodology (pp. 247268). West Sussex, Reino Unido: John Willey.

*Buckley-Walker, K., Crowe, T. P. y Caputi, P. (2013). Measuring Identity Processes in Family Relational Empowerment. International Journal of Mental Health and Addiction, 11(3), 358-368. https://doi.org/10.1007/s11469-012-9420-3

*Crittenden, N. y Ashkar, C. (2012). The SelfCharacterization Technique: Uses, Analysis, and Elaboration. En P. Caputi, L. L. Viney, B. M. Walker y N. Crittenden (Eds.), Personal Construct Methodology (pp. 109-128). West Sussex, Reino Unido: Willey.

*Cote, R. L. (1995). George Kelly: The Theory of Personal Constructs and His Contributions to Personality Theory. Recuperado de https://files.eric.ed.gov/fulltext/ED397368.pdf

Chang, M. F., Ng, S. E., Tien, A., Man, R. C. y Thayala, J. (2013). A Randomized Controlled Study to Explore the Effect of Life Story Review on Depression in Older Chinese in Singapore. Health \& Social Care in the Community, 21(5), 545-553. https://doi.org/10.1111/hsc.12043

*Denicolo, P. (2003). Elicitation Methods to Fit Different Purposes. En F. Fransella (Ed.), International Handbook of Personal Construct 
Psychology (pp. 123-133). West Sussex, Reino Unido: John Willey.

Di Fabio, A. y Bernaud, J. L. (2018). Narrative Interventions in Post-Modern Guidance and Career Counseling: A Review of Case Studies and Innovate Qualitative Approaches. Cham, Suiza: Springer.

Dun, C. D. (2017). Personal Narratives and SelfTransformation in Postindustrial Societies. Annual Review of Anthropology, 46, 65-80. https://doi.org/10.1146/annurev-anthro-102116041702

*Faccio, E., Cipolleta, S., Romaioli, D. y Ruiba, S. (2011). Control in Bulimic Experience at the Beginning and the End of Therapy. Personal Construct Theory \& Practice, 8, 24-37.

Farkas, D. y Orosz, G. (2013). The Link between EgoResiliency and Changes in Big Five Traits after Decisión Making: The case of Extraversión. Personality and individual differences, 55(4), 440445. https://doi.org/10.1016/j.paid.2013.04.003

*Feixas, G. y Gallinat, N. (1989). La técnica de rol fijo [Fix-Rol Technique]. Revista de psiquiatría $y$ Psicología humanista, 26, 70-81.

Feixas, G. y Villegas, M. (1991). Personal Construct Analysis of Authobiographical Texts: A Method Presentation and Case Illustration. International Journal of Personal Construct Psychology, 4, 3183. https://doi.org/10.1080/08936039108404761

Feixas, G., Monferrer, M. C. Geldschläger, H. y Ruiz, B. G. (2002). Sistema de categorías de contenido para codificar constructos personales [System of Thematic Content to Codex Personal Construct]. Revista de Psicología General y Aplicada: Revista de la Federación Española de Asociaciones de Psicología, 55(3), 337-348.
García-Martínez, J. (2012). Técnicas narrativas en psicoterapia [Narratives Techniques in Psychotherapy]. Madrid, España: Síntesis.

Gergen, K. (1996). Realidades y relaciones: aproximaciones a la construcción social [Realities and Relationships: Soundings in Social Constructionism]. Barcelona, España: Paidós.

*Green, B. (2004). Personal Construct Psychology and Content Analysis. Personal Construt Theory \& Practice, 1(3), 82-91.

*Green, B. (2016). An Application of Computerised Corpus Linguistics Techniques to Analyze Text A Personal Construct Theory Perspective. Personal Construct Theory \& Practice, 13, 1-12.

*Green, D. y Kirby-Turner, N. (1990). First Steps in Family Therapy: A Personal Construct Analysis. Journal of Family Therapy, 12(2), 139-154. https://doi.org/10.1046/j..1990.00379

*Hamad, E. y Lee, C. (2013). A Personal Construct Approach to Discovering the Transition Experience of Studying Abroad. Personal Construct Theory \& Practice, 10, 15-27.

*Hardison, H. G. y Neimeyer, R. A. (2007). Numbers and Narratives: Quantitative and Qualitative Convergence Across Constructivist Assessments. Journal of Constructivist Psychology, 20(4), 285308. https://doi.org/10.1080/10720530701503827

*Hardison, H. G. y Neimeyer, R. A. (2012). Assessment of Personal Constructs: Features and Functions of Constructivist Techniques. En P. Caputi, L. L. Viney, B. M. Walker y N. Crittenden (Eds.), Personal Construct Methodology (pp. 3-52). West Sussex, Reino Unido: John Willey.

*Hopkins, N. J. (2012). Is there a Typical Agoraphobic Core Structure? Personal Construct Theory \& Practice, 9, 19-27. 
*Jackson, S. R. (1988). Self-Characterization: Dimensions of Meaning. En F. Fransella y L. F. Thomas (Eds.), Experimenting with personal construct psychology (pp. 223-231). Londres, Reino Unido: Routledge.

*Kelly, G. A. (1955). Personal Construct Psychology Vol 1: Theory and personality. Londres, Reino Unido: Routledge.

Landfiel, A. W. (1971). Personal Construct Systems in Psychotherapy. Oxford, Reino Unido: Rand McNally.

Lindquist, K. A. y Barret, L. F. (2008). Emotional Complexity. En M. Lewis, J. M. Haviland-Jones y Barret L. F. (Eds.), Handbook of emotions (pp. 513-533). Nueva York, NY: Guilford Press.

*Lovenfosse, M. y Viney, L. L. (1999). Understanding and Helping Mothers of Children with "Special Needs" Using Personal Construct Group Work. Community Mental Health Journal, 35(5), 431442. https://doi.org/10.1023/A:1018782411768

Masini, C. (2015). Psicodrama. Reflexiones desde la práctica clínica en un Hospital de Día Psiquiátrico [Psychodrama. Reflections form the Clinic Practice in a Psychiatric Day Hospital]. Arteterapia. Papeles de arteterapia y educación artística para la inclusión social, 10, 153-164.

Mair, J. M. M. (1977). The community of Self. En D. Bannister (Ed.), New Perspectives in Personal Construct Theory. Nueva York, NY: Academic Press.

McAdams, D. P. (1993). The Stories we Live by: Personal Myths and the Making of the Self. Nueva York, NY: Guildford.

McAdams, D. P. (1994). Can Personality Change? Levels of Stability and Growth in Personality across the Lifespan. En T. Heatherton y J. Weinberger (Eds.), Can personality change? (pp. 299-213). Washington, DC: APA Books.
McLean, S. (2015). Individual Autonomy or Social Engagement? Adult Learners in Neoliberal Times. Adult Education Quarterly, 65(3), 196-214. https://doi.org/10.1177/0741713615573984

Moreno, J. L. (1995). Psicodrama [Psychodrama]. Buenos Aires, Argentina: Lumen.

Müller, K. W., Beutel, M. E., Wölfling, K. (2014). A Contribution to the Clinical Characterization of Internet Addiction in a Sample of Treatment Seekers: Validity of Assessment, Severity of Psychopathology, and Type Co-Morbidity. Comprehensive Psychiatry, 55(4), 770-777. https://doi.org/10.1016/j.comppsych.2014.01.010

Neimeyer, G. J. (1996). Evaluación Constructivista [Constructivist Assessment]. Barcelona, España: Paidos.

*Neimeyer, R. A., Ray, L., Hardison, H., Raina, K., Kelley, R. y Krantz, J. (2003). Fixed Role in a Fishbowl: Consultation-based Fixed Role Therapy as a Pedagogical Technique. Journal of Constructivist Psychology, 16(3), 249-271. https://doi.org/10.1080/10720530390209270

*Procaccia, R., Veronese, G. y Castiglioni, M. (2014). Self-characterization and Attachment Style: A Creative Method of Investigating Children's Construing. Journal of Constructivist Psychology, 27(3), 174-193. https://doi.org/10.1080/10720537. 2014.904701

Real Academia Española. (2014). Caracterizar. Diccionario de la lengua española [Dictionary of Spanish Language] (23 ${ }^{\text {a }}$ ed.). Recuperado de http://dle.rae.es/?id=7OpEEFy

*Reed, N., Winter, D., Schulz, J., Aslan, E., Soldevilla, J. M. y Kuzu, D. (2014). An Exemplary Life? A Personal Construct Analysis of the Autobiography of Rudolf Hoess, Commandant of Auschwitz. Journal of Constructivist Psychology, 27(4), 274288. https://doi.org/10.1080/10720537.2013.84921 4 
Spence, D. P. (1982). Narrative Truth and Historical Truth: Meaning and Interpretation in Psychoanalysis. Nueva York, NY: Norton.

Stephenson, G. M., Laszlo, J., Ehmann, B., Lefever, R. [Robert] M. H. y Lefever, R. [Robin]. (1997). Diaries of Significant Events: Socio-linguistic Correlates of Therapeutic Outcomes in Patients with Addiction Problems. Journal of Community \& Applied Social Psychology, 7, 389-411. https://doi.org/10.1002/(SICI)10991298(199712)7:5<389::AID-CASP434>3.0.CO;2$\mathrm{R}$

Traen, B. y Sorensen, D. (2008). A Qualitative Study of how Survivors of Sexual, Psychological, and Physical Abuse Manage Sexuality and Desire. Sexual and Relationship Therapy, 23(4), 377-391. https://doi.org/10.1080/14681990802385699

*Truneckova, D. y Viney, L. L. (2007). Evaluating Personal Construct Group with Troubled Adolescents. Journal of Counselling \& Development, 85(4), 450-460. https://doi.org/10.1002/j.1556-6678.2007.tb00613 .X

*Truneckova, D. y Viney, L. L. (2012). Personal Construct Psychology Model of School Counselling Delivery. British Journal of Guidance \& Counselling, 40(5), 431-448. https://doi.org/10. 1080/03069885.2012.718739

*Vall, B. y Botella, L. (2014). Narrative Assessment: Differences between Anxious and Depressed Patients. Narrative Inquiry, 24(1), 132-152. https://doi.org/10.1075/ni.24.1.07val

*Veronese, G., Procaccia, R. y Castiglioni, M. (2012). Attachment Styles and Construction of Self in a Clinical Group of Aerophobic: A Pilot Study. Psychological studies, 57(3), 303-309. https://doi.org/10.1007/s12646-011-0136-4
*Veronese, G., Procaccia, R., Romaioli, D., Barola, G. y Castiglioni, M. (2013). Psychopathological Organizations and Attachment Styles in Patients with Fears of Flying: A Case Study. The Open Psychology Journal, 6, 20-27. https://doi.org/10. 2174/1874350101306010020

*Veronese, G., Procaccia, R., Ruggiero, G. M., Sassaroli, S. y Castiglioni, M. (2015). Narcissim and Defending Self-Esteem. An Exploratory Study Base on Self-characterizations. The Open Psychology Journal, 2015, 8, 38-43. https://doi.org/10.2174/1874350101508010038

*Viney, L. L. y Caputi, P. (2012). Using Constructivistoriented Content Analysis Scales. En P. Caputi, L. L. Viney, B. M. Walker y N. Crittenden (Eds.), Personal Construct Methodology (pp. 223-246). West Sussex, Reino Unido: Willey.

*Winter, D. A. y Tschudi, F. (2015). Construing a "Perfect Knight": A Personal Construct Investigation of Mass Murder. Journal of Constructivist Psychology, 28(2), 139-151. https://doi.org/10.1080/10720537.2013.846837

*Winter. D. A., Gournay, K., Metcalfe, C. y Rossotti, N. (2006). Expanding Agoraphobics' Horizons: An Investigation of the Effectiveness of a Personal Construct Psychotherapy Intervention. Journal of Constructivist Psychology, 19, 1-29. https://doi.org/10.1080/10720530500311141 


\section{Apéndice A. Listado y clasificación de referencias incluidas en la revisión}

\begin{tabular}{|c|c|c|c|c|c|c|}
\hline Autor(es) & Año & Título del trabajo & Tip. & Met. & Ins. & An. \\
\hline Androutsopoulou & 2001 & $\begin{array}{l}\text { The Self-characterization as a Narrative Tool: Applications in } \\
\text { Therapy with Individuals and Families. }\end{array}$ & $\mathrm{E}$ & Q & $\mathrm{M}$ & $\mathrm{NC}$ \\
\hline Ashkar & 2015 & $\begin{array}{l}\text { Finding our many Selves: A Personal Construct Psychology } \\
\text { Approach to Positive Mental Health. }\end{array}$ & $E$ & Q & M & A \\
\hline $\begin{array}{l}\text { S. J. Bell y R. C. } \\
\text { Bell }\end{array}$ & 2008 & $\begin{array}{l}\text { An Illustration of Self Characterization in a Child's Drawing: The } \\
\text { Importance of Process. }\end{array}$ & $\mathrm{E}$ & Q & A & C \\
\hline $\begin{array}{l}\text { Blowers y } \\
\text { O'Connor }\end{array}$ & 1995 & $\begin{array}{l}\text { Construing Contexts: Problems and Prospects of George Kelly's } \\
\text { Personal Construct Psychology. }\end{array}$ & $\mathrm{T}$ & C & NC & NC \\
\hline Botella y Feixas & 1998 & La exploración del sistema de construcción del cliente. & $\mathrm{T}$ & Q & C & C \\
\hline $\begin{array}{l}\text { Buckley-Walker et } \\
\text { al. }\end{array}$ & 2013 & Measuring Identity Processes in Family Relational Empowerment. & $\mathrm{E}$ & $\widehat{M}$ & M & A \\
\hline $\begin{array}{l}\text { Crittenden y } \\
\text { Ashkar }\end{array}$ & 2012 & $\begin{array}{l}\text { The Self-Characterization Technique: Uses, Analysis and } \\
\text { Elaboration. }\end{array}$ & $\mathrm{T}$ & M & M & M \\
\hline Cote & 1995 & $\begin{array}{l}\text { George Kelly: The Theory of Personal Constructs and His } \\
\text { Contributions to Personality Theory. }\end{array}$ & $\mathrm{T}$ & Q & NC & NC \\
\hline Denicolo & 2003 & Elicitation Methods to fit Different Purposes. & $\mathrm{T}$ & Q & C & NC \\
\hline Faccio et al. & 2011 & $\begin{array}{l}\text { Control in Bulimic Experience at the Beginning and the End of } \\
\text { Therapy. }\end{array}$ & $E$ & Q & A & A \\
\hline Feixas y Gallinat & 1989 & La técnica de rol fijo & $\mathrm{E}$ & Q & C & NC \\
\hline B. Green & 2004 & Personal Construct Psychology and Content Analysis. & $\mathrm{T}$ & C & NC & A \\
\hline B. Green & 2016 & $\begin{array}{l}\text { An application of computerised corpus linguistics techniques to } \\
\text { analyze text - A personal construct theory perspective }\end{array}$ & $\mathrm{E}$ & Q & A & A \\
\hline $\begin{array}{l}\text { D. Green y } \\
\text { Kirby-Turner }\end{array}$ & 1990 & First steps in family therapy: A personal construct analysis & $\mathrm{E}$ & Q & A & A \\
\hline Hamad y Lee & 2013 & $\begin{array}{l}\text { A personal construct approach to discovering the transition } \\
\text { experience of studying abroad. }\end{array}$ & $\mathrm{E}$ & M & C & A \\
\hline $\begin{array}{l}\text { Hardison y R. A. } \\
\text { Neimeyer }\end{array}$ & 2007 & $\begin{array}{l}\text { Numbers and narratives: Quantitative and Qualitative } \\
\text { Convergence across Constructivist Assessments. }\end{array}$ & $\mathrm{E}$ & C & C & A \\
\hline $\begin{array}{l}\text { Hardison y R. A. } \\
\text { Neimeyer }\end{array}$ & 2012 & $\begin{array}{l}\text { Assessment of Personal Constructs: Features and Functions of } \\
\text { Constructivist Techniques. }\end{array}$ & $\mathrm{T}$ & Q & C & M \\
\hline Hopkins & 2012 & Is there a Typical Agoraphobic Core Structure? & $\mathrm{E}$ & Q & A & A \\
\hline Jackson & 1988 & Self-characterization: Dimensions of Meaning. & $\mathrm{T}$ & C & A & A \\
\hline Kelly & 1955 & Personal Construct Psychology Vol 1: Theory and Personality. & $\mathrm{T}$ & Q & C & C \\
\hline $\begin{array}{l}\text { Lovenfosse y } \\
\text { Viney }\end{array}$ & 1999 & $\begin{array}{l}\text { Understanding and helping mothers of children with "special } \\
\text { needs" using Personal Construct Group Work. }\end{array}$ & $\mathrm{E}$ & Q & C & A \\
\hline $\begin{array}{l}\text { R. A. Neimeyer et } \\
\text { al. }\end{array}$ & 2003 & $\begin{array}{l}\text { Fixed Role in a Fishbowl: Consultation-based Fixed Role Therapy } \\
\text { as a Pedagogical Technique. }\end{array}$ & $\mathrm{E}$ & Q & C & A \\
\hline Procaccia et al. & 2014 & $\begin{array}{l}\text { Self-characterization and Attachment Style: A Creative Method of } \\
\text { Investigating Children's Construing. }\end{array}$ & $\mathrm{E}$ & C & A & A \\
\hline Reed et al. & 2014 & $\begin{array}{l}\text { An Exemplary Life? A Personal Construct Analysis of the } \\
\text { Autobiography of Rudolf Hoess, Commandant of Auschwitz. }\end{array}$ & $\mathrm{E}$ & Q & A & A \\
\hline $\begin{array}{l}\text { Truneckova y } \\
\text { Viney }\end{array}$ & 2007 & Evaluating Personal Construct Group with Troubled Adolescents. & $\mathrm{E}$ & Q & C & A \\
\hline $\begin{array}{l}\text { Truneckova y } \\
\text { Viney }\end{array}$ & 2012 & $\begin{array}{l}\text { Personal Construct Psychology Model of School Counselling } \\
\text { Delivery. }\end{array}$ & $\mathrm{T}$ & Q & $\mathrm{NC}$ & NC \\
\hline Vall y Botella & 2014 & $\begin{array}{l}\text { Narrative Assessment: Differences between Anxious and } \\
\text { Depressed Patients. }\end{array}$ & $\mathrm{E}$ & C & C & A \\
\hline Veronese et al. & 2012 & $\begin{array}{l}\text { Attachment Styles and Construction of Self in a Clinical Group of } \\
\text { Aerophobic: A Pilot Study. }\end{array}$ & $\mathrm{E}$ & M & C & $A$ \\
\hline
\end{tabular}




\begin{tabular}{|c|c|c|c|c|c|c|}
\hline Autor(es) & Año & Título del trabajo & Tip. & Met. & Ins. & An. \\
\hline Veronese et al. & 2013 & $\begin{array}{l}\text { Psychopathological Organizations and Attachment Styles in } \\
\text { Patients with Fears of Flying: A Case Study. }\end{array}$ & $\mathrm{E}$ & $\mathrm{M}$ & $\mathrm{C}$ & A \\
\hline Veronese et al. & 2015 & $\begin{array}{l}\text { Narcissim and Defending Self-esteem. An Exploratory Study } \\
\text { Base on Self-Characterizations. }\end{array}$ & $\mathrm{E}$ & Q & C & A \\
\hline Viney y Caputi & 2012 & Using Constructivist-oriented Content Analysis Scales. & $\mathrm{T}$ & C & NC & A \\
\hline Winter et al. & 2006 & $\begin{array}{l}\text { Expanding Agoraphobics' Horizons: An Investigation of the } \\
\text { Effectiveness of a Personal Construct Psychotherapy } \\
\text { Intervention. }\end{array}$ & $\mathrm{E}$ & C & M & $\mathrm{NC}$ \\
\hline Winter y Tschudi & 2015 & $\begin{array}{l}\text { Construing a "Perfect Knight": A Personal Construct Investigation } \\
\text { of Mass Murder. }\end{array}$ & $E$ & $\mathrm{Q}$ & A & A \\
\hline
\end{tabular}

Nota. Tip. (tipología del estudio): T (Teóricos), E (Empíricos); Met. (Metodología): Q (Cualitativo), C (Cuantitativo), M (Mixta); Ins. (Instrucciones): C (Clásica), A (Alternativa), M (Mixta), NC (No Consta); An. (Análisis): C (Clásica), A (Alternativa), M (Mixta), NC (No Consta).

\title{
REVIEW OF SELF-CHARACTERIZATION: A CONSTRUCTIVE NARRATIVE TECHNIQUE
}

\author{
JAVIER GONZÁLEZ-ENCINAS ${ }^{1}$, LUIS ÁNGEL SAÚL ${ }^{1}$ Y \\ JESÚS GARCÍA-MARTÍNEZ ${ }^{2}$
}

\section{EXTENDED SUMMARY}

The self-characterization is a narrative technique generate in Kelly's personal construct theory (PCT; Kelly, 1955). Self-characterization, as a technique, pretend to explore how people structure the world, how anticipate the experience through this structure and where they are located. It differs from the other narrative techniques through elicited instructions and the assessment protocol. The classic instructions that Kelly propose say:

I want you to write a character sketch of (name of the person), just as if he were the principal character in a play. Write it as it might be written by a friend who knew him very intimately and very sympathetically, perhaps better than anyone ever really could know him. Be sure to write it in the third person. For example, star out by saying (name of the person) is... (Kelly, 1955, pp. 242)
The instructions were inspired by Moreno's psychodrama. Specifically, the author has to differentiate of the rest and put himself in the shoes of other. In the theatre representation it is opened a negotiation process where protagonist chose the ways and meaning that he or she goes to move on. For Kelly, the instructions request some more deep that the own physical appearance, trying to move away the author from a negative characterization, a judge vision and promoting a narrative sense of a perception of self.

Other meaning question about this technique is by analysis format. To assess self-characterization has been proposed a body of attitudes that the therapist should be keep. These attitudes were contemplated in different phases of protocol of analysis. The main therapeutic attitude was the credulous approach, where the principal meaning is which the author is telling. Other therapeutic attitudes like reflection against contexts are related to the new identifications of meanings through study of new locations of sentence in the text to discover new meaning; 
shifting emphasis, where try to preserve any bias that prevented to understand which the author is telling; restatement of the argument through therapist construct, that try to approach the meaning of therapist and client; professional subsuming of personal constructs that integrate the personal construct system of client into the therapist.

However therapeutic attitudes are important in the analysis process of self-characterization, there are other phases relate to extract data in the classic protocol of analysis. The main objective to explore is personal constructs. To do this, different phases are purpose like observation of organization, that try to know which safest construct are, collation of terms, that explore the repeated terms to see low elaborated constructs, or dimensional analysis, that allows to identify all constructs in self-characterization. As well, self-characterization pay attention in causeeffect sentences in thematic analysis phase, that helps to understand how client focus the therapeutic change. A traditional phase in any narrative analysis is the study of a topical areas in the text. In self-characterization all topics or themes are study in a couple of phases: observation of sequence and transition and analysis of contextual areas invoked by the protocol. In the observation of sequence and transition phase, the main objective is finding the transitions from topic to topic. This transition should be represented like tries to find balance. In analysis of contextual areas invoked by the protocol is studied the main areas where the client is secure to elaborate his personal construct system.

Following Kelly's approach, this technique helps to stablish clinical hypothesis in different therapeutic process and for upgrade the therapeutic alliance. Further, its utility was linked to fixed-role therapy, where try to give a new role for client, that he had to use in day by day, to extend or reduce the vision of its self or world around. But no systematic review or meta-analysis, which collects the main findings of the technique, has been found. It is present a review that offer a compilation about self-characterizations findings; it is analysed the researches and make differences between the instructions and the analysis protocols, and focus areas. To compare the works, it has had into account study typologies (theorical or empirical), the methodological strategies (qualitative, quantitative, and mix), the instruction and correction typologies (classic or alternative). The search has been made through five data bases: Psycinfo, ScienceDirect, PsicoDoc, WoS y PubPsyc, using the descriptors; self-characterización, self characterization or autocaracterización in Spanish language. This review is divided in four sections that give an answer about the planed objectives. First, it is exposed the researches taking account typology and methodological strategies. Second, it is portrayed the instruction and assessment format. Third, it is observed which are the analysis phases that are more frequently found. At last, it will be concluded which are the future lines, its implication in other areas, and the self-characterization limits.

The first conclusion that it was obtained in the theoretical works are that do not exist an only valid analysis format. The credulous approach and clinic utility were two aspect commons in these researches. The credulous approach treats with the text as if the narrative topic will be true. The clinic utility is related to the flexibility which the researcher can modify the instructions by therapeutic need. Further, social desirability is highlighted by its link with the instruction format since it can work as a possible bias. The empirical researches consider that self-characterization is useful when it is exploring the emotions, change ways in treatment, thematic content of personal construct, personal construct in specific populational group, fixed role therapy, or conversion into a repertory grid technique. Some difficulties were detected when the results were tried to homogenize because of instructions and analysis format disparity.

In the second objective, when it is exploring the instruction formats, three aspect appeared repeatedly: third person use, intimacy, and credulous approach. When it is exploring the correction strategies, the researchers put some interest into extract concrete data. However, the selfcharacterization was created apart from psychometric process, but some authors had tried, for example Jackson (1988).

The third objective served for discover the main phase in analysis protocol in the most of works. It was the exploration of personal construct, "dimensional analysis", where the researcher tries to identify and classify the thematic content of personal construct. The study of personal construct and its classification help to see the personal 
construct system and allows comparison with another constructivist technique.

In conclusion, most recent works are associated to research formulate at empirical and qualitative level. At qualitative level, the researches have been interested by clinical intervention, subjective description linked to psychopathologies, the relationship between psychological variables and thematic content of the construct in the selfcharacterization. The quantitative studies have been interested by data extraction through scales and result comparison with other techniques. The mix studies have been concerned by the exploration about construct associated to a concrete psychopathology.

The self-characterization does not precise a context, because of the text are created by the vision from another person. In this manner, the author has freedom for purpose a simple description, or a facts exposition. Some studies have been located using alternative analysis protocols. However, none of the proposals has had a great impact on an empirical level. But, the exploration of personal construct has been the main phase in the most of researches. The self-characterization focused in the study of the choice that a person makes, the construct that choose for him or herself, and not in the story or the ability for telling stories. Two difficulties were displayed in the review, the social desirability bias and homogenization data fault.

It becomes necessary a model of analysis that unified the essential phases of self-characterization protocol of analysis. An example of this model will contain the study of personal construct, the exploration about different topics in the text and their discontinues, identification and classification cause-effect sentences, and attributional style study. In the same manner, the exploration about different roles, and characters. This model could be an example about systematic process of self-characterization analysis. That would help to upgrade the status of the selfcharacterization and would allow the uses of different informatic program of analysis. The present review has the same limitations that a narrative review. However, the impossibility is highlighted about compare the conclusions. The potentiality of self-characterization is very wide, and it is considered that a systematic protocol may help to get concrete data that allow to replicate studies. 
